\title{
An improved Probability Assessment Model of Electric Vehicle Chargers influencing on Distribution Transformer
}

\author{
Fen Qin ${ }^{1, a}$, Liang Shen ${ }^{1, b}$, Wei Hu ${ }^{1, \mathrm{c}}$, Shuangtong Chen ${ }^{1, \mathrm{~d}}$, Fan $\mathrm{Ye}^{2, \mathrm{e}}$ and \\ Yushuang $\mathrm{Li}^{2, \mathrm{f}}$ \\ ${ }^{1}$ Yuhang Power Supply Company of State Grid Zhejiang Hangzhou Electric Power Company, \\ Hangzhou 311100, China; \\ ${ }^{2}$ State Key Laboratory of Power Transmission Equipment \& System Security and New Technology, \\ Chongqing University, Chongqing 400044, China \\ a415397022@qq.com, b871965882@qq.com, 529737265@qq.com, d451592205@qq.com, \\ e745328181@qq.com
}

Keywords: distribution transformer; electric vehicle chargers; harmonic current; loss-of-life Abstract. In this paper, the charging current harmonics of EV are analyzed based on the harmonically coupled admittance matrix of three-phase rectification charger. The harmonic current calculation formula of single and multiple EV chargers in the grid is derived, and subsequent a probability method to assess the top oil temperature, hotspot temperature, relative aging rate and loss-of-life of distribution transformer affected by the charging current harmonics of EV chargers is proposed using the harmonic loss factor to quantify the impact of harmonic current on transformer and combining the probabilistic simulation method of charging load for multiple types of EV.

\section{Introduction}

Electric vehicle (EV), as the most potential new energy vehicle which is environmental protection and energy saving, has become the main direction of the car's development of all countries. With the spreading of EV and construction of EV charging station, the scale of EV charging will have significant influence on the safety and economic operation of power grid, including decrease of the quality of power supply, increase of network loss, lower equipment utilization rate and so on ${ }^{[1]}$.The impact caused by EV chargers charging EV will Increase the pressures of Power generation equipments, transmission equipments and distribution equipments ${ }^{[2-4]}$, and it mainly influences the distribution equipments, such as transformer, cable and switch equipment. Specifically, In the long run, the access of EV chargers will speed up the aging of distribution transformer. And from a short-term perspective, charging load in peak which leads to the action of transformer overload protection, will cause power outage for some electric power users ${ }^{[3]}$. Therefore, we need to elaborate analysis the influence of EV chargers on the distribution transformer. Then it can provide guidance for selection of transformer in EV charging stations.

At home and abroad, For study about the influence of EV chargers on distribution transformer, no one has analyzed the differences in different charging methods (conventional, quick charging and battery changes), and they also doesn't taking into account the harmonic current generated in the power grid when the EV chargers charge EV. Under the same load level, when the distribution transformers supply power to the nonlinear load, EV chargers, as a typical harmonic source ${ }^{[5,6]}$, generating harmonic current which will further rise the hot spot temperature of distribution transformers winding. Based on the coupled harmonic admittance matrix which represents the relation between current harmonic and voltage harmonic outputted by EV chargers, This paper puts forward the current harmonic algorithm of EV chargers connected to power grid. Making use of harmonic loss factor to quantify life lost of transformers which is caused by the current harmonic of EV chargers, and combining with charging load probability simulation, this paper puts forward an improved probability assessment method of EV chargers influencing on distribution transformer. According the analysis on a charging station, the correctness of the proposed scheme is validated. 


\section{The Temperature of Distribution Transformer and It's Life Loss Under The Harmonic Produced by EV Chargers}

The charging current harmonic of EV chargers and its influence on the distribution transformer need to be determined when the charging load is known. As for oil-immersed self-cooling transformer, it can be known, according to IEEE C57.91-1995standard, that the rise of the transformer top oil temperature to ambient temperature $\theta_{\mathrm{TO}}$ is roughly proportional to the 0.8 power of total loss of transformer $P_{\mathrm{TL}}$. Based on the total loss $P_{\mathrm{TL}}$ of transformer on rated operating condition, and under charging load, the transformer top oil temperature is:

$$
\begin{aligned}
\theta_{\mathrm{TOa}} & =\theta_{\mathrm{TO}}+\theta_{\mathrm{a}}=\theta_{\mathrm{TOR}}\left(\frac{P_{\mathrm{TL}}}{P_{\mathrm{TLR}}}\right)^{r}+\theta_{\mathrm{a}} \\
& =\theta_{\mathrm{TOR}}\left(\frac{P_{\mathrm{LL}}+P_{\mathrm{NL}}}{P_{\mathrm{LLR}}+P_{\mathrm{NL}}}\right)^{0.8}+\theta_{\mathrm{a}}
\end{aligned}
$$

Where $\theta_{\mathrm{TOR}}$ is the rise of the transformer top oil temperature to ambient temperature under rated loss, $\theta_{\text {Toa }}$ is the top oil temperature, $\theta_{\mathrm{a}}$ is the ambient temperature, $P_{\mathrm{LL}}$ is the load loss of transformer under charging load, and $P_{\mathrm{LLR}}$ is the load loss of transformer under its rated operating conditions, $P_{\mathrm{NL}}$ is the no-load loss. $\mathrm{R}$ is the experience coefficient, and for oil-immersed self-cooling transformer $r=0.8$. Transformer loss includes copper loss, eddy current loss, stray loss and so on. In order to quantify the effects of the current harmonic produced by EV chargers, adoptting loss factor ${ }^{[7]}$ to determine the ratio of loss of winding eddy current and stray, under harmonic the load loss can be acquired as:

$$
\begin{aligned}
P_{\mathrm{LL}} & =I^{2} R_{\mathrm{dc}}+F_{\mathrm{HL}} P_{\mathrm{EC}}+F_{\mathrm{HL}}^{\prime} P_{\mathrm{OSL}} \\
& =\sum_{h=1}^{H} I_{h}^{2} R_{\mathrm{dc}}+F_{\mathrm{HL}} P_{\mathrm{EC}}+F_{\mathrm{HL}}^{\prime} P_{\mathrm{OSL}}
\end{aligned}
$$

The two harmonic loss factors can be expressed as follows:

$$
F_{\mathrm{HL}}=\frac{\sum_{h=1}^{H}\left(\frac{I_{h}}{I_{1}}\right)^{2} h^{2}}{\sum_{h=1}^{H}\left(\frac{I_{h}}{I_{1}}\right)^{2}}, F_{\mathrm{HL}}^{\prime}=\frac{\sum_{h=1}^{H}\left(\frac{I_{h}}{I_{1}}\right)^{2} h^{0.8}}{\sum_{h=1}^{H}\left(\frac{I_{h}}{I_{1}}\right)^{2}}
$$

Considering that the harmonic current have influence on eddy current loss significantly, Stray loss can be ignored for facilitating computations ${ }^{[8]}$. And then the rise of winding hot-spot temperature $\theta_{\mathrm{g}}$ to the top oil temperature is proportional to the 0.8 power of transformer load loss $P_{\mathrm{TL}}$, winding hot-spot temperature can be get as follows:

$$
\begin{aligned}
\theta_{\mathrm{h}} & =\theta_{\mathrm{g}}+\theta_{\mathrm{TOa}}=\theta_{\mathrm{gR}}\left(\frac{P_{\mathrm{LL}}}{P_{\mathrm{LLR}}}\right)^{r}+\theta_{\mathrm{TOa}} \\
& =\theta_{\mathrm{gR}}\left(\frac{1+F_{\mathrm{HL}} P_{\mathrm{ECR}}}{1+P_{\mathrm{ECR}}}\right)^{r}+\theta_{\mathrm{TOa}}
\end{aligned}
$$

Where $\theta_{\mathrm{h}}$ is the hot-spot temperature, $\theta_{\mathrm{gR}}$ is the rise of winding hot-spot temperature to the top oil temperature under rated current, $P_{\mathrm{LLR}}$ and $P_{\mathrm{ECR}}$ are load loss and eddy current loss of winding respectively under rating state. Because of the uneven distribution of temperature of transformer, the partial degradation of transformer is the most serious when it is running on the highest temperature. For the transformer with non-thermal modification or thermal modification felt paper. Relative aging rate $V$, which is based on hot-spot temperature of winding, has a value of 1 when the hot spot temperature is $98^{\circ} \mathrm{C}$ or $110^{\circ} \mathrm{C}$. Then the relative aging rate of distribution transformer is as follows,

$$
V=\left\{\begin{array}{l}
2^{\frac{\theta_{h}-98}{6}}, \text { non-thermal modification } \\
e^{\frac{15000}{110+273}-\frac{15000}{\theta_{h}+273}}, \text { thermal modification }
\end{array}\right.
$$


In the time interval $[\mathrm{t} 1, \mathrm{t} 2]$, the life lost $\mathrm{L}$ of transformer is,

$$
L=\sum_{n=1}^{N} V_{n} \times t_{n}
$$

Where $n$ is the corresponding serial number for [t1, t2] which has been divided into multiple time interval, $\mathrm{N}$ is the total number of time intervals, and $V_{\mathrm{n}}$ is the relative aging rate of nth time interval, $t_{\mathrm{n}}$ is the length of nth time interval.

\section{Analysis of Harmonic Produced by EV Chargers And Evaluation of Life Loss of Distribution Transformer}

As a typical harmonic load, the equivalent current of harmonic analysis of EV chargers is as shown in figure $1^{[5]}$. Using coupled harmonic admittance matrix can accurately make each harmonic voltage and each current harmonic relate to each other. Based on three-phase uncontrolled rectifier, Literature [16] derives coupled admittance matrix of harmonic of EV chargers, and then it converts the nonlinear charging current of time domain into linear of frequency domain,

$$
\boldsymbol{I}_{\mathrm{Ch}}=\boldsymbol{Y}^{+} \boldsymbol{U}_{k}+\boldsymbol{Y}^{-} \boldsymbol{U}_{k}^{*}
$$

That is,

$$
\begin{gathered}
{\left[\begin{array}{c}
\dot{I}_{\mathrm{Cl}} \\
\dot{I}_{\mathrm{C} 5} \\
\dot{I}_{\mathrm{C} 7} \\
\vdots \\
\dot{I}_{\mathrm{CH}}
\end{array}\right]=\left[\begin{array}{ccccc}
Y_{1,1}^{+} & Y_{1,5}^{+} & Y_{1,7}^{+} & \cdots & Y_{1, K}^{+} \\
Y_{5,1}^{+} & Y_{5,5}^{+} & Y_{5,7}^{+} & \cdots & Y_{5, K}^{+} \\
Y_{7,1}^{+} & Y_{7,5}^{+} & Y_{7,7}^{+} & \cdots & Y_{7, K}^{+} \\
\vdots & \vdots & \vdots & \ddots & \vdots \\
Y_{H, 1}^{+} & Y_{H, 5}^{+} & Y_{H, 7}^{+} & \cdots & Y_{H, K}^{+}
\end{array}\right]\left[\begin{array}{c}
U_{1} \angle 0 \\
U_{5} \angle \varphi_{5} \\
U_{7} \angle \varphi_{7} \\
\vdots \\
U_{K} \angle \varphi_{K}
\end{array}\right]+} \\
{\left[\begin{array}{ccccc}
Y_{1,1}^{-} & Y_{1,5}^{-} & Y_{1,7}^{-} & \cdots & Y_{1, K}^{-} \\
Y_{5,1}^{-} & Y_{5,5}^{-} & Y_{5,7}^{-} & \cdots & Y_{5, K}^{-} \\
Y_{7,1}^{-} & Y_{7,5}^{-} & Y_{7,7}^{-} & \cdots & Y_{7, K}^{-} \\
\vdots & \vdots & \vdots & \ddots & \vdots \\
Y_{H, 1}^{-} & Y_{H, 5}^{-} & Y_{H, 7}^{-} & \cdots & Y_{H, K}^{-}
\end{array}\right]\left[\begin{array}{c}
U_{1} \angle 0 \\
U_{5} \angle-\varphi_{5} \\
U_{7} \angle-\varphi_{7} \\
\vdots \\
U_{K} \angle-\varphi_{K}
\end{array}\right]}
\end{gathered}
$$

Where $\boldsymbol{I}_{\mathrm{Ch}}$ is the Charging current harmonic vector, $\boldsymbol{U}_{\mathrm{k}}$ and $\boldsymbol{U}_{\mathrm{k}}{ }^{*}$ are the voltage harmonic and its conjugate vector, $\boldsymbol{Y}^{+}$and $\boldsymbol{Y}^{-}$is the coupled admittance matrix of harmonic, $U_{\mathrm{k}}$ and $\varphi_{\mathrm{k}}$ are the kth harmonic voltage RMS and phase, $\mathrm{H}$ is the highest current harmonic frequency, and $\mathrm{K}$ is the highest voltage harmonic frequency. The coupling relationship between output current harmonic of EV chargers and terminal voltage relate to each other through the $\boldsymbol{Y}^{+}$and $\boldsymbol{Y}^{-}$. And the elements of admittance matrix relate to the parameters $R_{\mathrm{f}} 、 L_{\mathrm{f}} 、 C$ and $R$ in the harmonic analysis equivalent circuit, as shown figure 1a. According to literature [9], Different charging power should only change the DC equivalent resistance R of EV charger.

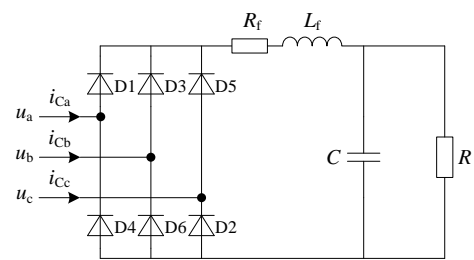

(a) the equivalent current of harmonic analysis of EV charger

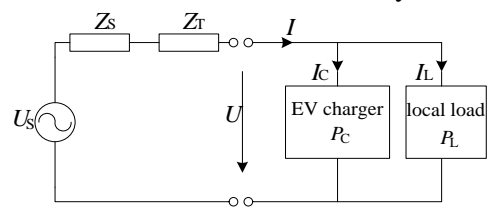

(b) system diagram of EV charger in distribution grid

Fig. 1 Schematic diagram for harmonic current calculation of EV charger in distribution grid 
Considering that the coupled admittance matrix $\boldsymbol{Y}^{-}$is far less than $\boldsymbol{Y}^{+}$on the amplitude, and fundamental voltage of power grid is much greater than each voltage harmonic, the elements at the first column of $\boldsymbol{Y}^{-}$in (8) are retained, and other elements of $\boldsymbol{Y}^{-}$can be ignored. As shown in figure 1b, impedance load model is used to represent the conventional local load in the low voltage side of distribution transformer. After EV charging load and local load all have been connected to distribution transformer, the current in low voltage side of distribution transformer is as follows:

$$
\begin{aligned}
{\left[\begin{array}{c}
\dot{I}_{1} \\
\dot{I}_{5} \\
\dot{I}_{7} \\
\vdots \\
\dot{I}_{H}
\end{array}\right] } & =\left[\begin{array}{ccccc}
Y_{\mathrm{L} 1} & 0 & 0 & \cdots & 0 \\
0 & Y_{\mathrm{L} 5} & 0 & \cdots & 0 \\
0 & 0 & Y_{\mathrm{L} 7} & \cdots & 0 \\
\vdots & \vdots & \vdots & \ddots & \vdots \\
0 & 0 & 0 & \cdots & Y_{\mathrm{LH}}
\end{array}\right]\left[\begin{array}{c}
U_{1} \angle 0 \\
U_{5} \angle \varphi_{5} \\
U_{7} \angle \varphi_{7} \\
\vdots \\
U_{K} \angle \varphi_{K}
\end{array}\right]+ \\
& {\left[\begin{array}{ccccc}
Y_{1,1}^{+}+Y_{1,1}^{-} & Y_{1,5}^{+} & Y_{1,7}^{+} & \cdots & Y_{1, K}^{+} \\
Y_{5,1}^{+}+Y_{5,1}^{-} & Y_{5,5}^{+} & Y_{5,7}^{+} & \cdots & Y_{5, K}^{+} \\
Y_{7,1}^{+}+Y_{7,1}^{-} & Y_{7,5}^{+} & Y_{7,7}^{+} & \cdots & Y_{7, K}^{+} \\
\vdots & \vdots & \vdots & \ddots & \vdots \\
Y_{H, 1}^{+}+Y_{H, 1}^{-} & Y_{H, 5}^{+} & Y_{H, 7}^{+} & \cdots & Y_{H, K}^{+}
\end{array}\right]\left[\begin{array}{c}
U_{1} \angle 0 \\
U_{5} \angle \varphi_{5} \\
U_{7} \angle \varphi_{7} \\
\vdots \\
U_{K} \angle \varphi_{K}
\end{array}\right] } \\
& =\boldsymbol{I}_{\mathrm{L} h}+\boldsymbol{I}_{\mathrm{Ch}}=\left(\boldsymbol{Y}_{\mathrm{L}}+\boldsymbol{Y}_{\mathrm{C}}\right) \boldsymbol{U}_{k}=\boldsymbol{Y}_{\mathrm{LC}} \boldsymbol{U}_{k}
\end{aligned}
$$

Where $\boldsymbol{I}_{\mathrm{Lh}}$ is the harmonic vectors of local load, $\boldsymbol{Y}_{\mathrm{C}}$ and $\boldsymbol{Y}_{\mathrm{L}}$ are the harmonic admittance matrix of EV charger and local load respectively. each harmonic of local load is uncoupling, so $\boldsymbol{Y}_{\mathrm{L}}$ is a diagonal matrix which is consist of $Y_{\mathrm{L} h}=1 / R_{\mathrm{L}}-\mathrm{j} 1 /\left(h \omega L_{\mathrm{L}}\right)$, where $R_{\mathrm{L}} 、 L_{\mathrm{L}}$ and $\omega$ are the resistance, inductance, and grid rated angular frequency of local load. From figure $1 \mathrm{~b}$, voltage balance equation of each harmonic can be get as,

$$
\begin{aligned}
\boldsymbol{U}_{k} & =\boldsymbol{U}_{\mathrm{S} k}-\left(\boldsymbol{Z}_{\mathrm{S} k}+\boldsymbol{Z}_{\mathrm{T} k}\right)\left(\boldsymbol{I}_{\mathrm{L} h}+\boldsymbol{I}_{\mathrm{Ch}}\right) \\
& =\boldsymbol{U}_{\mathrm{S} k}-\boldsymbol{Z}_{k} \boldsymbol{I}_{h}
\end{aligned}
$$

For a distribution network which connects $m$ sets of EV chargers, If node 1 of the distribution network is the main source node and node 2 to $m+1$ connect with $m$ sets of $E V$ chargers, the current moving to the ith harmonic source is,

$$
\boldsymbol{I}_{h}^{i}=\left(\boldsymbol{Y}_{\mathrm{L}}^{i}+\boldsymbol{Y}_{\mathrm{C}}^{i}\right) \boldsymbol{U}_{k}^{i}
$$

That is,

$$
\begin{aligned}
{\left[\begin{array}{c}
\boldsymbol{I}_{h}^{2} \\
\boldsymbol{I}_{h}^{3} \\
\vdots \\
\boldsymbol{I}_{h}^{m+1}
\end{array}\right] } & =\left[\begin{array}{cccc}
\boldsymbol{Y}_{\mathrm{L}}^{2}+\boldsymbol{Y}_{\mathrm{C}}^{2} & 0 & \cdots & 0 \\
0 & \boldsymbol{Y}_{\mathrm{L}}^{3}+\boldsymbol{Y}_{\mathrm{C}}^{3} & \cdots & 0 \\
\vdots & \vdots & \ddots & \vdots \\
0 & 0 & \cdots & \boldsymbol{Y}_{\mathrm{L}}^{m+1}+\boldsymbol{Y}_{\mathrm{C}}^{m+1}
\end{array}\right]\left[\begin{array}{c}
\boldsymbol{U}_{k}^{2} \\
\boldsymbol{U}_{k}^{3} \\
\vdots \\
\boldsymbol{U}_{k}^{m+1}
\end{array}\right]
\end{aligned}
$$

Fundamental current and each harmonic current of power grid are produced by main source and $\mathrm{m}$ sets of EV chargers, while main source only injects fundamental current into power grid when its voltage only contains fundamental component. Making use of node impedance matrix between each harmonic source, the nodal voltage equation of $\mathrm{m}$ sets of EV chargers access point can be get as follows,

$$
\left[\begin{array}{c}
\boldsymbol{U}_{k}^{2} \\
\boldsymbol{U}_{k}^{3} \\
\vdots \\
\boldsymbol{U}_{k}^{m+1}
\end{array}\right]=\left[\begin{array}{c}
\boldsymbol{Z}_{k}^{21} \\
\boldsymbol{Z}_{k}^{31} \\
\vdots \\
\boldsymbol{Z}_{k}^{(m+1) 1}
\end{array}\right] \boldsymbol{I}_{S h}-\left[\begin{array}{cccc}
\boldsymbol{Z}_{k}^{22} & \boldsymbol{Z}_{k}^{23} & \cdots & \boldsymbol{Z}_{k}^{2(m+1)} \\
\boldsymbol{Z}_{k}^{32} & \boldsymbol{Z}_{k}^{33} & \cdots & 0 \\
\vdots & \vdots & \ddots & \vdots \\
\boldsymbol{Z}_{k}^{(m+1) 2} & \boldsymbol{Z}_{k}^{(m+1) 3} & \cdots & \boldsymbol{Z}_{k}^{(m+1)(m+1)}
\end{array}\right]\left[\begin{array}{c}
\boldsymbol{I}_{h}^{2} \\
\boldsymbol{I}_{h}^{3} \\
\vdots \\
\boldsymbol{I}_{h}^{m+1}
\end{array}\right]
$$

For single and multi-machine system, jointing (9) and (10), (12) and (13) respectively, the voltage vector of charger and local load can be get as,

$$
\boldsymbol{U}_{k}=\left(\boldsymbol{E}+\boldsymbol{Z}_{k} \boldsymbol{Y}_{\mathrm{LC}}\right)^{-1} \boldsymbol{U}_{\mathrm{Sk}}
$$

Where $\boldsymbol{E}$ is the unit matrix of $K \times K$ order. Substituting (14) into (12), each harmonic current of the 
low voltage side of distribution transformer under different EV charging power can be get.

The size of EV charging power will change the DC resistance of equivalent circuit in figure 1a, influence the admittance matrix $\boldsymbol{Y}_{\mathrm{LC}}$ of charge load and other local load and the each harmonic current $\boldsymbol{I}_{\mathrm{h}}$ of load connected with distribution transformer. The harmonic current generated by EV charging will lead the top oil temperature and hot-spot temperature of distribution transformer to change with the size of it's load, and then decide the life loss of transformer through the relative thermal aging rate. Therefore, combining simulation of EV charging load, harmonic current analysis of EV charger with life loss calculation of distribution transformer, the life evaluation process of transformer can be get.

\section{Conclusions}

This paper analyses the charging current harmonics of EV based on the harmonically coupled admittance matrix of three-phase rectification charger. The harmonic current calculation formula of single and multiple EV chargers in the grid is derived, and subsequent a probability method to assess the top oil temperature, hotspot temperature, relative aging rate and loss-of-life of distribution transformer affected by the charging current harmonics of EV chargers is proposed using the harmonic loss factor to quantify the impact of harmonic current on transformer and combining the probabilistic simulation method of charging load for multiple types of EV.

\section{References}

[1] Zhao Junhua, Wen Fushuan, Yang Aimin, et al. Impacts of electric vehicles on power systems as well as the associated dispatching and control problem[J]. Automation of Electric Power Systems, 2011, 35(14): 2-10, 29

[2] Ge Wenjie, Huang Mei, Zhang Weige. Economic operation analysis of the electric vehicle charging station[J]. Transactions of China Electrotechnical Society, 2013, 28(2): 15-21

[3] Sexauer J M, McBee K D, Bloch K A. Applications of probability model to analyze the effects of electric vehicle chargers on distribution transformers[J]. IEEE Transactions on Power Systems, 2013, 28(2): 847-854

[4] Chen Liangliang, Zhang Hao, Ni Feng, et al. Present situation and development trend for construction of electric vehicle energy supply infrastructure[J]. Automation of Electric Power Systems, 2011, 35(14): 11-17

[5] Zhang Qian, Han Weijian, Yu Jihui, et al. Simulation model of electric vehicle charging station and the harmonic analysis on power grid[J]. Transactions of China Electrotechnical Society, 2012, 27(2): 159-164

[6] Zhao Wei, Meng Jinling, Chen Ruimin, et al. Accurate measurement and traceability method of electrical energy for charging and discharging devices of electric vehicles[J]. Automation of Electric Power Systems, 2013, 37(11): 113-118

[7] Yildirim D, Fuchs E F. Measured transformer derating and comparison with harmonic loss factor (FHL) approach[J]. IEEE Transactions on Power Delivery, 2000, 15(1): 186-191

[8] IEEE Std C57.110-2008. IEEE Recommended Practice for Establishing Liquid-Filled and Dry-Type and Distribution Transformer Capability When Supplying Nonsinusoidal Load Currents[S]. New York: The Institute of Electrical and Electronics Engineers, 2008

[9] Zhou N, Wang J, Wang Q, et al. Capacity calculation of shunt active power filters for electric vehicle charging stations based on harmonic parameter estimation and analytical modeling[J]. Energies, 2014, 7(2014): 5425-5443. 